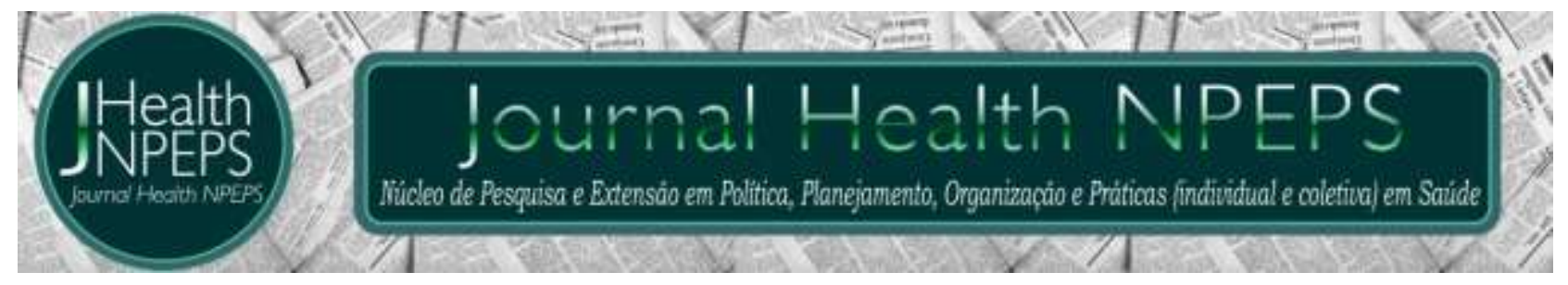

http://dx.doi.org/10.30681/25261010311122

ARTIGO ORIGINAL

\title{
Respostas cardiorrespiratórias e percepção subjetiva do esforço de hemiparéticos submetidos à prática de exergames
}

\section{Cardiorrespiratory responses and subjective perception of the effort in hemiparetics after exergames practice}

\section{Respuestas cardiorrespiratorias y percepción subjetiva del esfuerzo de hemipareticos después de la práctica de exergames}

\section{Luciana Maria de Morais Martins Soares ${ }^{1}$, Leilane Cândido Mariz Moreira², Wagner Irineu Medeiros de Souza ${ }^{3}$}

\section{RESUMO}

Objetivo: avaliar as respostas cardiorrespiratórias e percepção subjetiva do esforço de pacientes hemiparéticos submetidos à prática dos jogos de vídeo do XBOX Kinect. Método: estudo de casos múltiplos com abordagem quantitativa, com avaliação da frequência cardíaca, frequência respiratória, saturação de oxigênio, pressão arterial e percepção do esforço durante prática do jogo Your Shape Fitness Evolved $2012^{\circledR}$ cinco e dez minutos após o término da atividade, sendo considerados também os valores de frequência cardíaca máxima e de treino para fornecer mais segurança a pesquisa. Resultados: a amostra foi composta por dois sujeitos que apresentaram respostas adequadas a prática dos jogos ativos de vídeo game. Para o sujeito 1 a atividade foi considerada como leve e para o sujeito 2 uma atividade moderada. Conclusão: evidenciou-se a necessidade de mais estudos científicos para esclarecer os efeitos desses jogos sobre os diversos sistemas e a necessidade de profissionais habilitados a utilização desses jogos para fins terapêuticos.

Descritores: Paresia; Jogos de vídeo; Aptidão Cardiorrespiratória.

\section{ABSTRACT}

${ }^{1}$ Fisioterapeuta. Doutora em Evolução Humana: Antropologia Física e Forense. Professora no Centro Universitário de João Pessoa (UNIPE) e Uninassau João Pessoa. E mail: luciana momaso@hotmail.com ORCID ID: https://orcid.org/0000-0002-6559-9558 Autor principal - Endereço para correspondência: BR 230, Km22, Água Fria CEP: 58053-000, João Pessoa, Paraíba, Brasil.

${ }^{2}$ Fisioterapeuta. Centro Educacional de Ensino Superior de Patos (CESPE). Patos, Paraíba, Brasil. E-mail: leilanefisioterapia@gmail.com ORCID ID: https://orcid.org/0000-0002-9701-2774

${ }^{3}$ Fisioterapeuta. Especialização em Fisioterapia Cardiorrespiratória pelo Centro Universitário de João Pessoa (UNIPE) e em Preceptoria no SUS pelo Instituto Sírio-Libanês de Ensino e Pesquisa - IEP/HSL. Centro Educacional de Ensino Superior de Patos (CESPE). Patos, Paraíba, Brasil. E-mail: wagneririneu@hotmail.com ORCID ID: https://orcid.org/0000-0002-9547-0384 
Objective: to evaluate the cardiorespiratory responses and subjective perception of the effort of hemiparetic patients submitted to XBOX Kinect video games. Method: multiple case study with quantitative approach, with evaluation of heart rate, respiratory rate, oxygen saturation, blood pressure and perception of effort during practice of the game Your Shape Fitness Evolved 2012® five and ten minutes after the end of the activity, being also considered the values of maximum heart rate and training to provide more safety to the research. Results: the sample consisted of two subjects who presented adequate responses to the practice of active video game games. For subject 1 the activity was considered as mild and for subject 2 a moderate activity. Conclusion: the need for more scientific studies to clarify the effects of these games on the different systems and the need for professionals qualified to use these games for therapeutic purposes was evidenced.

Descriptors: Paresis; Video Games; Cardiorespiratory Fitness.

\section{RESUMEN}

Objetivo: evaluar las respuestas cardiorrespiratorias y percepción subjetiva del esfuerzo de pacientes hemiparéticos despúes de la práctica de los juegos de vídeo del XBOX Kinect. Método: Estudio de casos múltiples con abordaje cuantitativo, con evaluación de la frecuencia cardíaca, respiratoria, saturación de oxígeno, presión arterial y percepción del esfuerzo durante la práctica del juego Your Shape Fitness Evolved 2012 $₫$ después de cinco y diez minutos del término de la actividad. Se consideran también los valores de frecuencia cardiaca máxima y de entrenamiento para proporcionar más seguridad a la investigación. Resultados: la muestra fue compuesta por dos sujetos, éstos presentaron respuestas adecuadas a la práctica de los juegos activos de video. Para el sujeto 1 la actividad fue considerada como leve y para el sujeto 2 una actividad moderada. Conclusión: se evidenció la necesidad de más estudios científicos para aclarar los efectos de estos juegos sobre los diversos sistemas y la necesidad de profesionales habilitados para la utilización de esos juegos para fines terapéuticos.

Descriptores: Paresia; Juegos de vídeo; Capacidad Cardiovascular.

\section{INTRODUÇÃO}

O acidente vascular encefálico caracteriza-se por lesão focal ou global do encéfalo em decorrência da interrupção ou alteração significativa do fluxo sanguíneo cerebral, que afeta as habilidades motoras, cognitivas, sensoriais, comportamentais e perceptivas, podendo ser temporárias ou permanentes. Quando se ajusta o número de casos à idade tem-se uma estimativa de 108 casos por 100.000 habitantes, porém esses dados podem variar quando se leva em consideração os métodos utilizados, país em estudo e situação demográfica da população. A taxa de mortalidade aos trinta dias é cerca de $8,5 \%$ e aos doze meses de $30,9 \%$, onde o risco de recorrência após um ano é de aproximadamente $15,5 \%{ }^{1-3}$. 
No que concerne ao conjunto de possíveis manifestações clínicas apresentadas por um indivíduo após um AVE, é possível verificar alterações cardiovasculares, estando essas caracterizadas por mudança no ritmo cardiorrespiratório, na tensão dos vasos arteriais, na formação de coágulos nos vasos venosos dos membros inferiores, podendo interferir na circulação pulmonar e posteriormente levar a uma embolia pulmonar ${ }^{1}$.

O comprometimento de um hemicorpo é a manifestação mais comum após o AVE, podendo apresentar-se como hemiplegia, onde há ausência de movimentação, ou, hemiparesia, onde se observa redução da atividade e força muscular, dificultando a transferência de peso sobre o lado comprometido e gerando assimetria corporal, as quais interferem na manutenção do controle postural e estabilidade, que são elementos necessários a realização dos movimentos. No primeiro ano, a recuperação das habilidades motoras é mais evidente, e dessa maneira, após esse tempo as alterações motoras podem se tornar definitivas ${ }^{2,3}$.

O termo exergames é usado para definir a junção dos videogames à prática de atividade física, por propiciar a interação de todo o corpo humano, se tornando assim uma nova ferramenta contra o sedentarismo atualmente estabelecido ${ }^{4,5}$. No que concerne ao uso terapêutico dos exergames pela fisioterapia, inclusive com os indivíduos acometidos pelo $\mathrm{AVE}$, foram relatados diversos benefícios, como, por exemplo, melhora na coordenação, equilíbrio, força, amplitude de movimento tanto para os MMSS quanto os MMIl e, por conseguinte, na marcha ${ }^{6}$.

Ainda, deve-se destacar que esse tipo de atividade, por sua dinamicidade (ambiente interativo, sociável e de fácil aprendizagem) torna-se estimulante para o jogador/paciente, no entanto, evidencia-se que esse recurso deve ser usado como um coadjuvante ao tratamento convencional, tendo a função de auxiliar, manter e/ ou prolongar os efeitos dos recursos cinesioterapêuticos ${ }^{5,6}$.

Mesmo com a grande utilização dos exergames, as pesquisas a respeito das repercussões destes nos indivíduos que os praticam são escassas, e nos remete a um mundo de dúvidas a respeito do real dispêndio de energia e incrementos que há sobre as funções fisiológicas. Diante do exposto, o objetivo deste trabalho foi avaliar as respostas cardiorrespiratórias e percepção subjetiva do esforço de hemiparéticos submetidos à prática dos jogos de vídeo do XBOX Kinect.

\section{MÉTODO}


A presente pesquisa consiste em um estudo de casos múltiplos com abordagem quantitativa, desenvolvida em uma clínica-escola de fisioterapia de uma instituição privada de ensino superior do estado da Paraíba.

Esse estudo foi encaminhado à Plataforma Brasil, avaliado e aprovado pelo Comitê de Ética em Pesquisa em Seres Humanos do Centro Universitário de João Pessoa UNIPE (CAAE $n^{\circ}$ 35053914.6.0000.5181) que emitiu um parecer comprovando a seriedade da pesquisa e a concordância da mesma com as diretrizes e normas da Resolução 466/2012 do Conselho Nacional de Saúde/Ministério da Saúde.

A população do estudo foi composta por hemiparéticos após episódio de AVE, cuja amostragem pode ser considerada não probabilística e obtida por acessibilidade, mediante a assinatura do Termo de Consentimento Livre e Esclarecido - TCLE e a observância aos critérios de inclusão e exclusão estabelecidos para o presente estudo.

Como critérios de inclusão na pesquisa foram considerados pré-requisitos iniciais: 1) ter idade igual ou superior a 50 anos; 2) Apresentar hemiparesia em estado crônico, ou seja, superior a 6 meses devido à AVE isquêmico ou hemorrágico devidamente diagnosticado por laudo médico; 3) Não estar praticando nenhuma modalidade de exercício físico; 4) Deambular sem auxílio de dispositivos; 5) apresentar boa acuidade visual; 6) assinar o Termo de Consentimento Livre e Esclarecido - TCLE.

Como critérios de exclusão na pesquisa foram considerados: 1) usuários que apresentem notável rebaixamento cognitivo que o impossibilite compreender e responder adequadamente a entrevista e sua efetiva participação na atividade; 2) apresentar doenças crônico-degenerativas descompensadas; 3) apresentar dislipidemia; 4) apresentar problema ósseo ou articular que possa ser agravado com exercício físico; 5) apresentar episódios importantes de vertigem e labirintite.

Foram convidados a participar da pesquisa 22 pacientes acometidos por AVE com idades entre 50 e 80 anos, cadastrados nos serviços de atendimento fisioterapêutico da clínica escola. Desses, somente três indivíduos compareceram a avaliação, sendo excluído um por não apresentar movimento voluntário no membro superior, conforme fora estabelecido nos critérios do estudo.

Em contato feito por telefone, os participantes foram orientados a não consumir bebidas alcoólicas e/ou tabaco nos três dias que antecederam as coletas de dados, a não realizar atividade física forçada, a hidratar-se adequadamente, e dormir em média de 6 
a 8 horas, como também a apresentar no dia da avaliação inicial todos os exames que os mesmos haviam realizado recentemente. Ao final, restaram dois sujeitos participantes do estudo caracterizados a seguir e nomeados como sujeito 1 e sujeito 2.

Foram utilizados os seguintes instrumentos para coleta de dados: 1) Ficha de avaliação sociodemográfica e de condição clínica, com questões relacionadas à identificação como: nome, idade, sexo, estado civil, profissão, endereço, telefone, tempo transcorrido do episódio de AVE e tipo, assim como dados essenciais para a segurança da prática da atividade física, como história de dor referida no peito, braço ou mandíbula, condição osteomioarticular, pressão arterial, frequência cardíaca, respiratória e saturação de oxigênio; 2) Questionário de Prontidão para Atividade Física PAR-Q, para identificar os indivíduos que apresentam possíveis riscos cardiovasculares e/ou fatores de risco que possam limitar ou contraindicar a realização da atividade física7 $^{7}$ e, 3) A escala de percepção subjetiva do esforço através da Escala de Borg modificada $^{8}$, uma escala de 10 pontos que aproveita as melhores características da escala anterior e que melhor traduzem as sensações subjetivas do esforço, sendo elas a alteração da ventilação, utilização do sistema anaeróbico, força e dor.

O protocolo de avaliação dos sujeitos constou de três encontros:

1) Primeiro, destinado à avaliação através do questionário sociodemográfico e do questionário de prontidão para atividade física, além da análise dos exames apresentados;

2) Segundo, destinado ao conhecimento e prática com o console $X-B O X^{\circledR}$ Kinect $^{\circledR}$ e jogo Your Shape Fitness Evolved $2012^{\circledR}$, cujo protocolo foi organizado com: atividades prioritariamente realizadas com membros superiores, atividades prioritariamente realizadas com membros inferiores e atividades de movimentação global, incluindo saltos verticais, através dos minijogos kick it, pump it, hu lala, wall breaker, stomp it, $e$ humana (aging with grace), o último minijogo está subdividido em seis rotinas, onde a $1^{\text {a }}$ e $4^{\mathrm{a}}, 2^{\mathrm{a}}$ e $5^{\mathrm{a}}, 3^{\mathrm{a}}$ e $6^{\mathrm{a}}$ são iguais. Utilizou-se o tempo aproximado de uma sessão terapêutica, ou seja, 60 minutos, respeitando a sequência: aquecimento (10min.), alongamento (10min.), treino de modalidades motoras específicas (30min.) e relaxamento ou descontração (10min.).

3) Terceiro, verificação dos sinais vitais (SSVV) e percepção subjetiva do esforço, que ocorreram no período de repouso, ou seja, após 10 minutos aguardando a avaliação 
em sedestação; após a prática de cada minijogo; e, 5/10 minutos após a prática geral do exercício com os jogos

A pressão arterial (PA) foi medida através de um esfigmomanômetro aneroide e um estetoscópio da marca Premium ${ }^{\circledR}$ ambos fabricados no ano de 2011; a Frequência Cardíaca (FC), através do uso de um frequencímetro da marca OREGON ${ }^{\circledR}$, fabricado em 2013; a Frequência Respiratória (FR), acompanhada com o auxílio de um relógio, sendo observado o número de incursões respiratórias produzidas em um minuto e a Saturação Periférica de Oxigênio $\left(\mathrm{SpO}_{2}\right)$, acompanhada com o auxílio de um oxímetro da marca CONTEC $^{\circledR}$, modelo CMS50D, fabricado em 2013.

Utilizou-se o cálculo para estimar a FC máx, ou seja, a frequência desenvolvida durante a prática de atividade que exija um esforço máximo, através da fórmula: 220 idade, que é largamente utilizada nos estudos em que a análise da $\mathrm{FC}_{\text {máx. se faz }}$ necessária9.

Com intuito de prover mais segurança e controle ao trabalho, calculou-se a

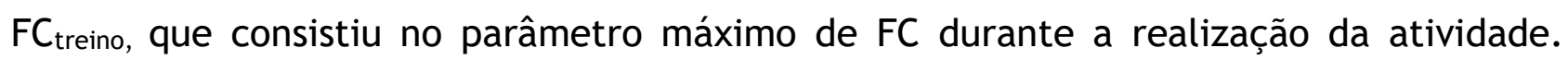
Permitiu-se um limite de FC que caracterizasse a atividade física como moderada, ou seja, dentro da margem de $64-77 \%$ da $\mathrm{FC}_{\text {máx. }}$, conforme recomendações do American College of Sports Medicine - $\mathrm{ACSM}^{10}$. Portanto, se utilizou a fórmula desenvolvida por Karvonen e colaboradores em 1957, onde: $\mathrm{FC}_{\text {treino }}=\mathrm{FC}_{\text {repouso }}+\%$ zona que se deseja trabalhar ( $\left.F C_{\text {máx. }}-F_{\text {repouso }}\right)$. Sendo que, caso esse limite fosse alcançado ou superado a atividade seria interrompida.

Ao final do procedimento de coleta e identificação dos dados, os mesmos foram armazenados em uma planilha do Microsoft Excel ${ }^{\circledR}$, sendo posteriormente analisados através de estatística descritiva simples.

\section{RESULTADOS}

O Sujeito 1, gênero masculino, 53 anos, casado, fumante, atuou profissionalmente como policial militar e caminhoneiro. O episódio de AVE ocorreu em fevereiro de 2014, sendo devidamente classificado como isquêmico. O mesmo relatou na avaliação inicial, não sentir dor no peito, braço ou mandíbula, ou nas estruturas osteomioarticulares. Na avaliação dos exames apresentados, no hemograma completo 
não se verificou alterações nos níveis de glicose, triglicérides, colesterol e frações (HDL, LDL e VLDL), tendo os mesmos sido realizados recentemente.

No ecocardiograma do sujeito 1 , detectou-se a presença de forame oval patente com shunt esquerdo e direito, sendo imediatamente solicitada avaliação cardiovascular do paciente, na qual foi obtida a liberação para participação na pesquisa. Faz uso de Losartana 50mg, Sinvastantina 20mg, Hidroclorotiaziada 25mg, Somalgin Cardio $100 \mathrm{mg}$ e Clopidogrel 75mg. A FC máx. calculada foi de $167 \mathrm{bpm}$ e a $F C_{\text {treino } 77 \%}=152 \mathrm{bpm}$.

Durante a prática dos minijogos, o sujeito 1 apresentou respostas cardiorrespiratórias adequadas durante a prática dos minijogos, sendo requisitada pelo mesmo uma $\mathrm{FC}$ média de $113 \pm 3 \mathrm{bpm}$, correspondendo a uma faixa de $59 \%$ da $\mathrm{FC}_{\text {máx. }}$, enquadrando-o na faixa de atividade leve que vai de $50<64 \%$ da $\mathrm{FC}_{\text {máx. }}$, segundo o $A C S M^{13}$. Sendo que, o pico foi $118 \mathrm{bpm}$ durante a sexta rotina, o que corresponde a uma faixa de $61 \%$ da $\mathrm{FC}_{\text {máx. }}$

Tabela 1 - Resultados da avaliação do sujeito 1.

\begin{tabular}{|c|c|c|c|c|c|c|c|c|c|c|c|c|c|c|}
\hline \multirow[t]{2}{*}{ VAR. } & \multirow[t]{2}{*}{ REP } & \multirow{2}{*}{$\begin{array}{l}\text { KIC } \\
\text { T IT }\end{array}$} & \multirow{2}{*}{$\begin{array}{l}\text { PU } \\
\text { MP } \\
\text { IT }\end{array}$} & \multirow{2}{*}{$\begin{array}{c}\text { HU } \\
\text { LAL } \\
\text { A }\end{array}$} & \multirow{2}{*}{$\begin{array}{c}\text { WALL } \\
\text { BREAK } \\
\text { ER }\end{array}$} & \multirow{2}{*}{$\begin{array}{c}\text { STO } \\
\text { MP } \\
\text { IT }\end{array}$} & \multicolumn{6}{|c|}{ HUMANA- AGING WICH GRACE } & \multirow{2}{*}{$\begin{array}{c}5 \\
\text { MIN }\end{array}$} & \multirow{2}{*}{$\begin{array}{r}10 \\
\text { MIN }\end{array}$} \\
\hline & & & & & & & R1 & R2 & R3 & R4 & R5 & R6 & & \\
\hline FC & 102 & 109 & 109 & 110 & 110 & 114 & 110 & 111 & 117 & 114 & 117 & 118 & 99 & 99 \\
\hline BORG & 0 & 0 & 1 & 0 & 0 & 0 & 0 & 0 & 0 & 0 & c & 0 & 0 & 0 \\
\hline SPO2 & 98 & 98 & 97 & 98 & 97 & 97 & 96 & 96 & 97 & 96 & 96 & 97 & 99 & 98 \\
\hline PA & $\begin{array}{c}110 / \\
70 \\
25\end{array}$ & - & - & - & - & - & - & - & - & - & - & $\begin{array}{c}120 / \\
70 \\
27\end{array}$ & $\begin{array}{l}120 \\
170 \\
27\end{array}$ & $\begin{array}{l}110 \\
170 \\
25\end{array}$ \\
\hline
\end{tabular}

Legenda: VAR= variáveis; $R E P$ = repouso; $R$ = rotina(sequência de movimentos apresentados no jogo).

0 sujeito 2, gênero masculino, 72 anos, casado, atuou profissionalmente como vendedor ambulante de calçados, tendo ocorrido o episódio de AVE em dezembro de 2013, sendo diagnosticado como do tipo isquêmico. Ao exame físico, o mesmo não relatou a presença de dores mandibulares, no peito, braço ou nas estruturas osteomioarticulares. Nos exames apresentados, a exemplo do hemograma completo, não se verificaram alterações nos níveis plasmáticos de glicose, triglicérides, colesterol e suas frações (HDL, LDL e VLDL). Além disso, também não houve presença de alterações no eletrocardiograma e ecocardiograma. 0 mesmo não faz uso de nenhum medicamento. Entretanto, o mesmo suspeita ser portador de labirintite, porém sem grandes repercussões sobre o equilíbrio. $A \mathrm{FC}_{\text {máx. }}$ calculada foi de $148 \mathrm{bpm}$ e a $\mathrm{FC}$ treino $77 \%=134$ bpm. 
O sujeito 2 obteve respostas cardiorrespiratórias adequadas durante a prática dos minijogos, obtendo uma média de $113 \pm 7 \mathrm{bpm}$ durante as atividades, o que

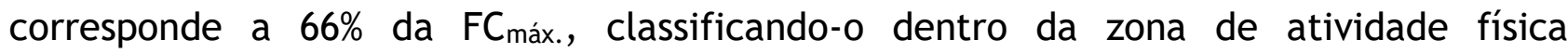

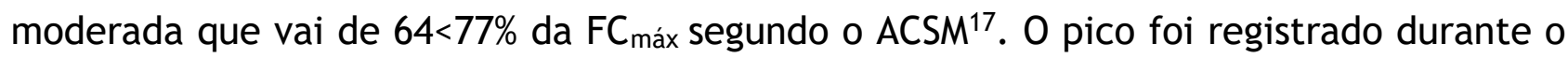
minijogo pump it e na segunda rotina alcançando $120 \mathrm{bpm}$, solicitando uma FC

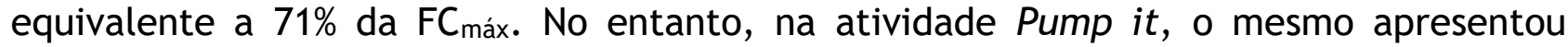
percepção subjetiva do esforço 5 , sendo solicitada pelo participante a interrupção da atividade. Durante a pausa, o sujeito foi instruído a fazer exercícios respiratórios e hidratar-se até que o cansaço relatado diminuísse e o mesmo sentir-se apto a prosseguir.

Tabela 2 - Resultados da avaliação do sujeito 2.

\begin{tabular}{|c|c|c|c|c|c|c|c|c|c|c|c|c|c|c|}
\hline \multirow[t]{2}{*}{ VAR. } & \multirow[t]{2}{*}{ REP } & \multirow{2}{*}{$\begin{array}{c}\text { KICT } \\
\text { IT }\end{array}$} & \multirow{2}{*}{$\begin{array}{l}\text { PUMP } \\
\text { IT }\end{array}$} & \multirow{2}{*}{$\begin{array}{c}\text { HU } \\
\text { LAL } \\
\text { A }\end{array}$} & \multirow{2}{*}{$\begin{array}{c}\text { WALL } \\
\text { BREAK } \\
\text { ER }\end{array}$} & \multirow{2}{*}{$\begin{array}{c}\text { STOMP } \\
\text { IT }\end{array}$} & \multicolumn{6}{|c|}{ HUMANA- AGING WICH GRACE } & \multirow{2}{*}{$\begin{array}{c}5 \\
\text { MIN }\end{array}$} & \multirow{2}{*}{$\begin{array}{c}10 \\
\text { MIN }\end{array}$} \\
\hline & & & & & & & R1 & R2 & R3 & R4 & R5 & R6 & & \\
\hline FC & 88 & 100 & 120 & 117 & 119 & 115 & 118 & 120 & 119 & 115 & 105 & 110 & 99 & 99 \\
\hline BORG & 0 & 1 & 5 & 3 & 2 & 1 & 1 & 2 & 3 & 1 & 1 & 2 & 0 & 0 \\
\hline SPO2 & 97 & 96 & 97 & 98 & 98 & 97 & 97 & 97 & 98 & 96 & 98 & 98 & 99 & 98 \\
\hline PA & $\begin{array}{c}110 / \\
70\end{array}$ & - & - & - & - & - & - & - & - & - & - & $\begin{array}{l}100 \\
160\end{array}$ & $\begin{array}{l}100 \\
/ 60\end{array}$ & $\begin{array}{c}90 / \\
60\end{array}$ \\
\hline FR & 18 & - & - & - & - & - & - & - & - & - & - & 22 & 20 & 20 \\
\hline
\end{tabular}

Legenda: VAR= variáveis; REP = repouso; $R$ = rotina (sequência de movimentos apresentados no jogo).

\section{DISCUSSÃO}

Estudos envolvendo a prática de exergames por pessoas acometidas por AVE comumente trazem a perspectiva de sua utilização em distúrbios de controle postural, equilíbrio estático e dinâmico ou funcionalidade do membro superior parético ${ }^{11-15}$.

$\mathrm{Na}$ revisão sistemática realizada por Ruivo ${ }^{16}$ para avaliar se os exergames são eficazes na melhoria de vários parâmetros de saúde em adultos/idosos, em particular para a reabilitação cardíaca, verificou-se que a prática resulta em intensidades de jogabilidade que poderiam ser classificadas entre leve-moderada. Apesar da maioria dos estudos também apontarem para melhora do equilíbrio corporal, existem relatos sobre sua utilização para melhorar a aptidão cardiorrespiratória. Mesmo não havendo evidências que demonstrassem sua utilização no coletivo de pessoas acometidas por AVE, existem razões para acreditar que os exergames podem ser importantes para abordar outras temas relacionados à saúde e bem-estar desta população. 
Dentro da perspectiva da avaliação das funções cardiorrespiratórias, Silva et al ${ }^{17}$ estudaram vinte e sete adultos com Síndrome de Down (SD) que foram alocados aleatoriamente em um grupo experimental $(n=14)$ ou grupo controle $(n=13)$. Os participantes do grupo experimental completaram um programa de exercícios de 2 meses com base na prática de exergame proporcionado pelo console do Nintendo Wii, com jogos de treinamento para resistência aeróbica, equilíbrio e força isométrica. Os resultados obtidos sugerem que o exercício baseado no Wii pode ser uma ferramenta eficaz para melhorar a aptidão física, a mobilidade funcional e a proficiência motora de adultos com SD, incluindo medidas cruciais como capacidade aeróbica e força dos membros inferiores.

Em estudo realizado por Falcade et $\mathrm{al}^{18}$, utilizando o Your Shape Fitness Evolved $^{\circledR} 2010$, com 30 indivíduos do sexo masculino, jovens e saudáveis com média de idade de $23,1 \pm 1,8$ anos, observaram-se valores médios de $145 \pm 21,1 \mathrm{bpm}$ com o uso do jogo, sendo a prática do exergames, considerada, para este grupo como vigorosa.

Pereira ${ }^{19}$, em um estudo realizado junto a 18 voluntários de ambos os gêneros, com idade média de $22 \pm 1$ anos submetidos a um programa de exercitação física através do Xbox Kinect, verificou que novas tecnologias, com enfoque específico nos jogos virtuais e games ativos, impactam não somente o estado físico mas também emocional, especificamente quanto à motivação para a prática de exercícios e atividades físicas.

Comparando os achados da literatura com aqueles obtidos no presente estudo, verifica-se que embora os sujeitos participantes tenham sido acometidos por AVE isquêmico e o tempo de ocorrência do episódio tenha sido praticamente o mesmo, o nível de disfunção motora observado em ambos foi distinto. 0 sujeito 1 , apesar de mais jovem apresentou comprometimento motor mais significativo quando comparado com o sujeito 2. Além disso, observou-se que o sujeito 2 desenvolveu um Borg 5 no minijogo pump it, solicitando a parada da atividade, o que aparentemente reflete um resultado controverso.

Entretanto, identifica-se que o sujeito 1 manteve, durante a atividade, níveis de FC condizentes com uma atividade de intensidade leve. Em contrapartida, o sujeito 2, por apresentar déficits motores mais atenuados quanto comparado ao sujeito 1 obteve margens de exercitação física na faixa de intensidade moderada. Acredita-se assim, que a maior capacidade motora gera maior possibilidade em executar a tarefa com a 
ritmicidade exigida pelo jogo incrementando as respostas respiratórias, cardíacas e percepção de esforço.

Comparando-se os achados da presente pesquisa com os estudos supracitados pode-se observar que a intensidade da prática dos exergames sofre influência de outros fatores que precisam ser clarificados por estudos científicos. Apesar de não ter sido objeto de estudo, acredita-se que as limitações da função motora do sujeito 1 interferiram na execução do gestual global com perdas importantes na velocidade e precisão, gerando incremento nas respostas cardiovasculares.

Entre os 35 e 50 anos o indivíduo apresenta uma perda de força máxima de cerca de $6 \%$ a cada década, após essa idade há uma perda de cerca de $10 \%$ por década em decorrência da perda das fibras musculares, as fendas sinápticas se tornam mais profundas dificultando a propagação dos estímulos, repercutindo sobre a velocidade e precisão na realização dos movimentos. Comumente, são indicados exercícios físicos aeróbicos de modo a otimizar as funções cardiorrespiratórias de modo a minimizar os efeitos deletérios do processo de envelhecimento e da inatividade física provocada por acometimentos neurológicos incapacitantes ${ }^{16}$.

Nesse contexto, Ferraz et $\mathrm{al}^{20}$ compararam os efeitos do treinamento funcional, exercício em bicicleta e exergaming na funcionalidade de idosos ( $>=60$ anos de idade; $\mathrm{n}=62$ ) com doença de Parkinson. Os participantes foram aleatoriamente distribuidos em três grupos: grupo 1, que participou do treinamento funcional $(n=22)$; o grupo 2 , realizou exercício de bicicleta $(n=20)$ e o grupo 3 treinou com exergames da Kinect Adventures com o console do Xbox Kinect $(\mathrm{n}=20)$. Nesse estudo, verificou-se que oito semanas de exergaming pode melhorar a funcionalidade de idosos com DP, e que é possível verificar resultados físicos semelhantes em comparação com o treinamento funcional e o exercício de bicicleta, incrementando a funcionalidade dos participantes.

Pode-se sugerir que a idade e performance motora tenha um efeito interveniente nas respostas cardiovasculares, impactando assim na intensidade do jogo, porém, quando bem administrado e planejado pode trazer benefícios a esses indivíduos não somente dentro da perspectiva motora, mas também das funções cardiorrespiratórias e percepção de esforço pelos praticantes.

Quando se analisa a percepção subjetiva do esforço do sujeito 1, este não relatou em nenhum momento sentir-se cansado atribuindo valores iguais a 0 e 1 durante toda a atividade. Para o sujeito 2 obteve-se valores médios de 2 , o qual está relacionado 
com uma atividade de intensidade muito leve. Somente durante o minijogo pump it obteve-se valor de 5 , sendo este o máximo relatado e coincidindo com uma atividade de intensidade moderada, o que corresponde a uma pontuação 5-6 na escala do BORG segundo as recomendações do ACMS.

Analisando a pressão arterial, acredita-se a percepção de esforço apresentou-se dentro de níveis incapazes de gerar risco cardiorrespiratório, uma vez que não houve alteração importante da mesma, havendo um incremento de $10 \mathrm{mmHg}$ na pressão arterial sistólica (PAS) e manutenção da pressão arterial diastólica (PAD), voltando aos valores basais após dez minutos em repouso no sujeito 1 , e, redução de $10 \mathrm{mmHg}$ na PAS e PAD.

Resultados similares não foram reproduzidos em pessoas acometidas por disfunção neurológica, mas em estudo realizado com oito adultos jovens do gênero masculino, com idade média de 21ะ1,6 anos, sem restrições a atividade física e que não apresentavam experiência com os jogos de realidade virtual, foram registradas pressões arteriais após os jogos que variam de $110-140 \mathrm{mmHg}$ para PAS e de $60-80 \mathrm{mmHg}$ para a $\mathrm{PAD}^{21}$.

Saponisk et al $^{22}$ avaliaram a viabilidade, segurança e eficácia do uso de exergames em pessoas acometidas por AVE ( $n=22$, idade média $=61,3$ anos) inseridas em programas de intervenção com tempo médio total 364 minutos. Os mesmos concluíram que a mesma consiste em uma alternativa segura, viável e potencialmente efetiva. Constata-se assim, ao que tudo indica, que a prática de exergames após o AVE, nas condições estudadas e apresentadas, não configura risco às funções cardiorrespiratórias e percepção de esforço.

\section{CONCLUSÃO}

Como observado, a prática dos jogos ativos de vídeo game, por parte de pessoas acometidas por AVE, não gerou incremento junto as funções cardiorrespiratórias, mostrando-se ser uma atividade de baixo risco cardiovascular e adaptável à capacidade funcional do praticante.

Mesmo não sendo explícitos, os cuidados inerentes a prática dessa modalidade de exercitação física, especialmente junto às populações em condições neuromusculares mais fragilizadas, a exemplo dos idosos senis, devem ser discutidos, afim de aprofundar os conhecimentos acerca dos efeitos desses jogos junto aos diferentes sistemas, de 
modo a estabelecer normas, cuidados e precauções ao seu uso, bem como a necessidade de monitoramento mais rigoroso em prol da redução dos eventuais riscos.

Apesar desses achados iniciais não apontarem para risco eminente quanto a prática de jogos ativos de vídeo game por parte dessas populações, é fundamental a presença de pessoas qualificadas, capazes de reconhecer os sinais de fadiga e/ou instabilidades hemodinâmicas durante a utilização dos mesmos, especialmente para sua prática no âmbito domiciliar.

Os resultados obtidos são o ponta pé inicial para estudos nessa temática, e desta maneira, aponta-se como limitação do estudo o quantitativo de participantes, uma vez que um número maior de participantes poderia auxiliar a compreender com maior exatidão a problemática envolvida no presente estudo. Além disso, sugere-se a realização de mais estudos e elaboração de jogos específicos para aplicação dentro do âmbito da reabilitação.

\section{REFERÊNCIAS}

1. Ministério da Saúde (BR). Secretaria de Atenção à Saúde. Departamento de Ações Programáticas Estratégicas. Diretrizes de atenção à reabilitação da pessoa com acidente vascular cerebral. Brasília: Ministério da Saúde; 2013.

2. Rodrigues TA, Silva TSA, Esotico APCA, Heyn D, Naki IK, Battistella LR, et al. Acidente vascular encefálico agudo: reabilitação [internet]. 2012 [acesso: 18 set 2018]. Disponível

em: https://diretrizes.amb.org.br/_BibliotecaAntiga/acidente_vascular_encefalico_agud o_reabilitacao.pdf.

3. Terranova TT, Albieri FO, Almeida MD, Ayres DV, Cruz SF, Milazzotto MV, et al. Acidente vascular encefálico crônico: reabilitação [internet]. 2012 [acesso: 18 set 2018]. Disponível

em: https://diretrizes.amb.org.br/_BibliotecaAntiga/acidente_vascular_encefalico_croni co_reabilitacao.pdf.

4. Di Tore PA, Raiola G. Exergames e didattica delle attività Motorie e sportive. Eur J Sustain Dev. 2012; 1(2):221-8.

5. Monteiro Junior RS, Silva EB. Efetividade da reabilitação virtual no equilíbrio corporal e habilidades motoras de indivíduos com déficit neuromotor: uma revisão sistemática. Rev bras ativ fís saúde. 2012; 17(3):224-30. 
6. Cho $\mathrm{KH}$, Lee $\mathrm{KJ}$, Song $\mathrm{CH}$. Virtual-reality balance training with a video-game system improves dynamic balance in chronic stroke patients. Tohoku J Exp Med. 2012; 228(1):69-74.

7. Oliveira LLG, Maranhão Neto GDA, Farinatti PDTV. Validade do questionário de prontidão para a atividade física (par-q) em idosos. Rev bras cineantropom desempenho hum. 2007; 9(4): 366-71.

8. Borg GAV. Psychophysical bases of perceived exertion. Med Sci in Sports Exerc. 1982; 14(5):377-81.

9. American College of Sports Medicine. ACSM's Guidelines for Exercise Testing and Prescription. Baltimore: Lippincott Williams \& Wilkins; 2014.

10. Nelson ME, Rejeski WJ, Blair SN, Duncan PW, Judge JO, King AC, et al. Physical activity and public health in older adults: recommendation from the American College of Sports Medicine and the American Heart Association. Circulation. 2007; 116(9):1094-105.

11. Kim EK, Kang JH, Park JS. Jung BH. Clinical feasibility of interactive commercial Nintendo gaming for chronic stroke rehabilitation. J phys ther sci. 2012; 24(9):901-3.

12. Pavão SL, Sousa NVC, Oliveira CM, Castro PCG, Santos MCM. 0 ambiente virtual como interface na reabilitação pós-AVE: relato de caso. Fisioter em Mov. 2012; 26(2): 45562.

13. Morone G, Tramontano M, losa M, Shofany J, lemma A, Musicco M, et al. The efficacy of balance training with video game-based therapy in subacute stroke patients: a randomized controlled trial. Biomed Res Int. 2014; 1-6.

14. Silva WHS, Lopes GLB, Yano KM, Tavares NSA, Rego IAO, Cavalcanti FADC. Effect of a rehabilitation program using virtual reality for balance and functionality of chronic stroke patients. Motriz. 2015; 21(3):237-43.

15. Oliveira MPB, Ferreira DM, Silva JR, Silva AM, Lobato DFM, Kosour C, et al. Realidade virtual na função motora de membros inferiores pós-acidente vascular encefálico. Acta Fisiátrica. 2017; 23(3):135-9.

16. Ruivo JA. Exergames and cardiac rehabilitation: a review. J cardiopulm rehabil prev. 2014; 34(1):2-20.

17. Silva V, Campos C, Sá A, Cavadas M, Pinto J, Simões $P$, et al. Wii-based exercise program to improve physical fitness, motor proficiency and functional mobility in adults with Down syndrome. J Intellect Disabil Res. 2017; 61(8):755-65. 
18. Falcade AC, Baroncini LAV, Hanna EDA, Leitão MB, Schumann DR, Nanni FN, et al. Análise do consumo de oxigênio, da frequência cardíaca e equivalente metabólico obtidos através de um videogame ativo. Rev Insp. 2013; 5(6):20-4.

19. Pereira, F. C. Exergames: Fator motivacional para a prática de atividades físicas [dissertação]. Rio Claro: Universidade Estadual Paulista, Instituto de Biociências de Rio Claro (UNESP); 2016. 102 p.

20. Ferraz DD, Trippo KV, Duarte GP, Neto MG, Santos KOB, Oliveira Filho J. The effects of functional training, bicycle exercise and exergaming on walking capacity of elderly with Parkinson's disease: a pilot randomized controlled single-blinded trial. Arch Phys Med Rehabil. 2018; 99(5):826-33.

21. Perrier-Melo RJ, Brito-Gomes JL, Oliveira SFM, Costa MC. Respostas agudas da frequência cardíaca e da pressão arterial em uma sessão de jogos de vídeo game ativos em adultos saudáveis: um estudo piloto. Rev Ter Ocup. 2013; 24(3):256-6.

22. Saposnik G, Teasell R, Mamdani M, Hall J, Mcllroy W, Cheung D, et al. Effectiveness of virtual reality using Wii gaming technology in stroke rehabilitation: a pilot randomized clinical trial and proof of principle. Stroke. 2010; 41(7):1477-84.

Conflito de interesses: Os autores declaram não haver conflito de interesses.

\section{Participação dos autores:}

- Concepção: Soares LMMM, Moreira LCM.

- Desenvolvimento: Soares LMMM, Moreira LCM, Souza WIM.

- Redação e revisão: Soares LMMM.

Como citar este artigo: Soares LMMM, Moreira LCM, Souza WIM. Respostas cardiorrespiratórias e percepção subjetiva do esforço de hemiparéticos submetidos à prática de exergames. Journal Health NPEPS. 2018 jul-dez; 3(2): 492-505. 